BLS 35, No 1 2009. DOI: http://dx.doi.org/10.3765/bls.v35i1.3616

(published by the Berkeley Linguistics Society and the Linguistic Society of America)

\title{
Numeral Modifiers and Temporal Container Adverbials ${ }^{*}$
}

\author{
ANITA MITTWOCH \\ The Hebrew University of Jerusalem
}

\section{Introduction}

This paper is mainly concerned with restrictions on numeral modifiers (e.g more than/less than, at least/at most) inside in-adverbials, as in Jane ran a mile in 5 minutes. But before discussing these, the paper begins, in Section 1, by drawing attention to restrictions on these modifiers elsewhere, in contexts where a speaker assumes that the value of the numeral is low or high relative to a contextually shared norm. I shall term such assumptions 'evaluative'. The restrictions, it will be argued, are due to rhetorical considerations; the effect of inappropriate modifiers would go against the grain of the evaluative assumption. Section 2 will then proceed to in adverbials. These appear to measure the temporal length of extended telic eventualities, but their entailments and scalar implicatures go in the opposite direction from those of normal measure phrases. For reasons that will become clear in the discussion, such adverbials will be called 'container adverbials'. In Section 3 it will be shown that container adverbials are subject to a subset of the constraints discussed in Section 1. Section 4 will deal with some surprising data concerning the modifier at least, and Section 5 will extend the discussion to the use of in-adverbials in sentences like The train will arrive in ten minutes.

\section{Restrictions and Numeral Modifiers}

Unmodified numerals have an exhaustive ('exactly') scalar implicature (Grice 1975, Horn 1972). Modifiers on numerals yield fuzzy values. I shall deal mainly with the comparative modifiers more than and less than, and the superlative modifiers at least and at most. At least $n$ can be paraphrased as not less than $n / n$ or more, at most $n$ as not more than $n / n$ or less. Following Krifka (2006, 2007), I take superlative modifiers to have their locus in the speech act. At least $n$ implies that $\mathrm{n}$ is the lowest value for which the speaker can take full responsibility, with the possibility of a higher value left open. At most $n$ implies that $\mathrm{n}$ is the highest value for which the speaker can take full responsibility, with the possibility of a lower value (including 0) left open.

\footnotetext{
* Thanks to Edit Doron for helpful discussion and comments on an earlier version of the paper.
} 


\section{Anita Mittwoch}

In this section I note certain restrictions on these modifiers, connected to a speaker's assumptions about the quantity or amount of the entity that is measured by the numeral. A speaker who believes that the quantity is low would normally refrain from choosing an expression that points upward on the scale, i.e. that entails (in the case of more than) a higher value, or suggests the possibility of one (for at least); conversely, a speaker who believes that the quantity is high would refrain from choosing an expression that points downward. ${ }^{1}$

Let us assume a context in which you have been asked

How many boys are there in the choir?

You do not know the exact number of boys in the choir but you know that it is relatively small, certainly compared with the number of girls in the choir. You might make this explicit by saying at the outset Not many; alternatively, this may already be part of the common background. You know that the number is roughly ten, plus or minus a few. If you think that ten is the upper bound, (2a) would be appropriate but ( 2 b) would be doubtful: ${ }^{2}$

a. fewer than 10 , at most 10 , not more than 10 , not quite 10

b. almost 10

Suppose now that ten is the lower bound; suppose further that the person asking the question has no prior evaluative expectation about the number of boys in the choir. You begin by making it clear that the number is small. You couldn't just say

Not many; more than ten / at least ten / not less than ten

The modified numerals in (3) cry out for an adversative conjunction:

Not many, but more than ten / at least ten / not less than ten

If the fact that there are relatively few boys in the choir is part of the background, you could say a bit more than ten, but more than 10, at least ten no less than 10 would still be inappropriate.

\footnotetext{
${ }^{1}$ I suspect that the restrictions may turn out to be evaluative not only in the sense that relatively high/low values are assumed but in the sense of 'good' versus 'bad'. As I have not explored this matter more fully, I shall ignore it here.

${ }^{2}$ Almost has two meaning components, a negative or polar one (in our example 'not 10') and a proximal one ('close to 10'). Following Horn (2002) I take both to be entailments, but only the second to be asserted. Horn calls the polar entailment 'assertorially inert'; almost is "rhetorically oriented towards the positive component of its meaning".
} 


\section{Numeral Modifiers and Container Adverbials}

If you thought that the number is exactly 10, the modifiers in (4) would also be appropriate inasmuch as they are evaluative; they draw attention to the lowness of 10 on the scale: ${ }^{3}$

$$
\text { only } 10 \text {, no more than } 10
$$

Now let us assume you have been asked

$$
\text { How many girls are there in the choir? }
$$

Again, you do not know the exact number, but this time you know that there are relatively many, roughly fifty, and, if this is not part of the common ground, you start by saying $A$ lot. If you think fifty is the lower bound you can use the modifiers that were problematic in (3):

(6) (a bit) more than 50, no less than 50, at least 50

If you think 50 is the upper bound you cannot say (7a) but you can say (7b): ${ }^{4}$

a. fewer/less than 50, at most 50,

b. almost 50 , not quite 50 , a bit less than 50

In a context in which the speaker has no prior assumption that the value of the answer is relatively high or low, any of the modifiers could, of course, be chosen.

For the comparative and superlative modifiers the restrictions might be explained by the fact that the modified numerals would lack an upper bound; in theory, the values on the scale could be high/low enough to be on the wrong side of whatever line would be considered the border for low/high values according to the speaker's assumptions. I doubt, however, that this is a sufficient explanation for the restrictions. In practice these modifiers typically come with much more limited, though fuzzy, margins. Moreover, this explanation would not generalize to almost, since almost 10 is less rather than more than 10. I suggest that the main motivation for the restrictions is rhetorical; the inappropriate choices would be felt to weaken the evaluative assumption of a high/low value on the scale.

In support of this suggestion, note that the restrictions are not absolute. Consider Beth's response to Anne in (8) and (9).

(8) Anne: There aren't many boys in the choir this year, at most 10

Beth: At least 10, I would say.

\footnotetext{
${ }^{3}$ Nouwen (2008) points out that the preferred reading of no more than ten marbles is exactly ten marbles, attributing this reading to an implicature. He also points out that the reading "has an evaluative side-effect of expressing that ten marbles does not count as a lot." The observation is traced back to Stoffel (1894) and discussed in Jespersen (1949).

${ }^{4}$ Notice that not quite is not restricted; it appears both here and in (2a) above.
} 


\section{Anita Mittwoch}

(9) Anne: There are a lot of girls in the choir this year, at least 50.

Beth: At most 50 / less than 50 / not quite 50 / well, almost 50

Beth may well share Anne's belief that there are few boys and many girls in the choir, and differ from Anne only in her estimate of the numbers; her estimate is less extreme in terms of their shared assumptions than Anne's. This is sufficient to free up modifiers that we found to be inappropriate above. ${ }^{5}$ If the absence of an upper bound were the sole, or even main, reason for the restrictions, we would not expect the restrictions to be overridden so easily.

\section{Temporal Container Adverbials}

When we measure the length of a homogeneous eventuality, the measurement proceeds straightforwardly as in ordinary counting:

\section{Mary typed for an hour. ${ }^{6}$}

(10) entails that Mary typed for 10 minutes, half an hour, etc. (10) additionally implies that Mary typed for exactly an hour, no more than an hour. This is a scalar implicature, based on

the Maxim of Quantity: Say as much as you can and the Maxim of Quality: Do not say anything for which you lack evidence.

But if Mary, in fact, typed for more than an hour, (10) is not false.

Unlike homogeneous eventualities, extended telic events cannot be measured in this way. We appear to measure them by in-adverbials, as in

\section{Mary typed 10 pages in an hour.}

In fact we are not measuring the temporal length of Mary's typing directly in (11). What we are measuring is the interval which contains the event, or, conversely, into which the event fits (Krifka 1998, who calls such adverbials interval adverbials, Kearns 2003, Mittwoch 2010). The semantic and pragmatic relations are reversed. (11) does not entail that Mary typed 10 pages in half an hour. Instead it entails higher values:

She did it in an hour and a half, in 2 hours, etc.

These larger intervals would simply not be completely filled by the event. The scale for this type of measurement is a descending one. Again, (11) implies that

\footnotetext{
${ }^{5}$ Only is not amenable to forgoing its implication of a low value in this kind of context. If Beth thinks there are exactly 50 girls in the choir, she cannot respond with only 50.

${ }^{6}$ Strictly speaking, we measure the temporal trace of the event, rather than the event itself.
} 


\section{Numeral Modifiers and Container Adverbials}

she typed 10 pages in exactly an hour and, again, this is an implicature. If Mary did it in less than an hour (11) is not falsified. In other words, it is compatible with

\section{(13) Mary typed ten pages in 55 minutes. $^{7}$}

In that case, the hour mentioned in (11) would contain slack - time not filled by part of the event. The Maxim of Quality would stop you from saying (13) if you had insufficient evidence for its truth; the Maxim of Quantity would stop you from saying (12) if you knew (11) to be true. In terms of strength, (13) > (11) > (12). The strongest statement is the one that specifies the lowest point on the scale, the statement according to which the event totally fills the specified interval and is thus completed in the shortest time.

So far the entailments and implicatures involved in measurement along a descending scale appear to be a perfect mirror image of what happens when we measure along the more familiar rising scale. But there is also a significant difference: The entailed values of the rising scale can play a part in subsequent discourse. (10), repeated as (14a), can be continued as in (14b) or (14c):

a. Mary typed for an hour.

b. During the first twenty minutes she typed faster than for the rest of the hour.

c. Ten minutes before she finished she had to answer a phone call.

The entailed values of the descending scale cannot give rise to such continuations, since the larger intervals are empty of event content. The entailments seem to be backgrounded or 'inert', to borrow Horn's term (cf. Note 1) The effect of this difference is that the exhaustive ('exactly') implication is stronger for temporal container adverbials than it is for the durational adverbial in sentences like (10), or for values on rising scales in general.

As noted in Mittwoch (1980), these adverbials are inappropriate if the interval is considered to be relatively long, so that the speed of the event would be relatively slow:

Mary typed 10 pages in a (very) short / \#a (very) long time.

The construction is thus inherently evaluative. A speaker who reckons that an extended telic event was relatively slow would normally choose the alternative way of indirectly measuring the length of the event, the take construction, as in

\footnotetext{
${ }^{7}$ This is shown by the fact that a hearer cannot respond to (11) with (i) but only with (ii):

(i) $\quad \# N o /$ That's not true she did it in 55 minutes.

(ii) Actually, she did it in 55 minutes.
} 


\section{Anita Mittwoch}

It took Mary an hour to type three pages.

This construction works on a rising scale, and carries no evaluative implications. Thus

(17) It took her a very short / a very long time to type the paper.

If the speaker has no preconceived notion about the matter, either construction can be used.

\section{Numeral Modifiers in Container Adverbials}

In the absence of speakers' assumptions about Mary's typing for a particularly long or short time, (10) is compatible with any of the modifiers discussed in Section 1 .

Mary typed for more than / at least / less than / at most / almost / only an hour.

But for (11) we find the same restrictions that we observed in (3) above. The examples in (19) are unproblematic, while those in (20) are problematic out-of-theblue.

a. Mary typed 10 pages in less than / under an hour, in at most an hour, in no more than an hour.

b. Mary typed 10 pages in only an hour.

a. Mary typed 10 pages in more than / over an hour, at least an hour

b. Mary typed 10 pages in almost an hour. ${ }^{8}$

Note that modifiers which are weakeners for numerals on a rising scale serve to strengthen the speaker's claim in the good examples; conversely, modifiers that strengthen the claim for a rising scale would weaken it in the problematic ones. Instead of (20) one would normally use the take construction.

In view of what was said in the previous section about the evaluative implication of sentences with these adverbial, the above data are hardly surprising. They point in the same direction as (15) above. The adverbials are incompatible with any suggestion that the containing interval is relatively long so that the event would be slow.

We also saw in Section 1 that the implication of a low (or high) value can disappear in certain contexts. This applies here too:

\footnotetext{
${ }^{8}$ For almost $n$ on a descending scale there is the added complication that it is not always clear whether the value is above or below $\mathrm{n}$. Witness the ambiguity of The temperature is almost zero. Cf. Nouwen (2006).
} 
Numeral Modifiers and Container Adverbials

(21) Mary: I have typed ten pages in an hour.

Jane: According to my watch, you did it in more than an hour / in at least an hour and ten minutes.

Again, Jane's response does not mean that she denies absolutely that Mary's typing was relatively fast. (22) illustrates a straightforward comparison between two similar events, and need not carry any implication, let alone value judgement, about the speed of the performances:

(22) Today's pianist played the sonata in 27 minutes. The one I heard last week did it in at least 30 minutes.

In the next example, not only is the restriction on the numeral modifier in the adverbial overruled; additionally, the evaluative implication is flipped from fast to slow. This happens when the scalar value of the numeral in the object NP is weakened by a numeral modifier:

(23) a. Mary typed at most / less than / only ten pages in more than an hour.

b. Mary typed at most / less than / only ten pages in at least an hour.

c. Mary typed at most / less than / only ten pages in almost an hour.

The modifier in the adverbial reinforces what is already implied by the one in the object: Mary was slow. If the modifiers are reversed, the implication is that Mary was super-quick:

(24) Mary typed more than/ at least / almost ten pages in less than / at most / only one hours.

Roger Schwarzschild (p.c.) has suggested that the problem with the examples in (20) is the fact, noted in the previous section, that they are entailed by (11), the same sentence without a numeral modifier, and that they are therefore not just less informative than (11), but, strictly speaking, vacuous. (Mittwoch 2010). This would also explain the data in (15), repeated below for convenience:

Mary typed 10 pages in a (very) short / \#long time.

Any sentence with a container adverbial would entail the same sentence with $a$ long time replacing the temporal specification in the adverbial. The same is obviously not true for a short time.

Nevertheless it seems to me that this cannot be the whole explanation for the restrictions. For one thing, our reaction to a violation of the restriction is very different from our reaction to an inappropriate modifier on a rising scale. Compare (25a) below, one of the options from (20), with (25b), one of the options from (18): 


\section{Anita Mittwoch}

(25) a. Mary typed 10 pages in more than an hour

b. Mary typed for less than an hour

with (25b) said in a context in which it is known that she typed for an hourwhich entails that she typed for less than an hour. (25b) would be felt to be grossly misleading in this context; in asserting this entailment the speaker has subtracted part of the typing event. In (25a) the entailment from the interval specified in the container adverbial to a larger interval does not affect the quantity of the event. As suggested in the previous section, this kind of entailment is backgrounded, since the entailed higher value is, literally, 'uneventful'. (25a) is felt to be odd but neither misleading nor glaringly vacuous. Its oddness is of the kind that we encountered in Section 1. This would imply that the entailment to higher values is not computed; the implication of speed is not felt to follow from the entailment. Rather, this evaluative implication would be a built-in part of the meaning of container adverbials in neutral contexts, possibly a presupposition.

Furthermore, if we accept a semantic explanation for the problem with (20), we shall, again, be at a loss to account for examples like (21), (22), and (23), in which the restrictions on modifiers are inoperative. If the problem belongs to whatever branch of pragmatics will be able to tackle evaluative meaning, it should be easier to account for these counterexamples.

To conclude this section, I add examples showing that the restrictions discussed above apply to another descending scale. The first line of Beth's answers to Anne's questions in (26) is appropriate, the second is not. ${ }^{9}$

(26) Anne: How many bricks are sufficient to build the wall?

Beth: At most / less than / only 100

At least / more than / almost 100.

By contrast, all the answers would be appropriate if Anne's questions had been (27), which involves a rising scale:

How many bricks are needed to build the wall?

\section{More on at least}

On a rising scale at least has the potential of strengthening an utterance by pointing to the possibility of a higher value, while at most weakens by pointing to a lower value. I have shown that on a descending scale, at most strengthens (Example (19a)), and at least is normally inappropriate; it cannot serve to weaken, since this would conflict with the normal (out-of-the-blue) implication of sentences with container adverbials. The exceptions noted in connection with (21), (22), and (23) involve particular contexts, the first two of which are of a type that we also encountered in Section 0.

\footnotetext{
${ }^{9}$ The example is adapted from Beck and Rullmann (1999), cited in Fox and Hackl (2006).
} 


\section{Numeral Modifiers and Container Adverbials}

In this section I shall present further cases - and more surprising ones - where at least modifies a numeral situated on a descending scale. In most of the examples that follow both at least and at most could be used - with no difference in meaning. Consider

(28) My stuff will fit into at least the $80 \mathrm{~cm}$. case, perhaps even into the smaller one.

(29) (Context: Cooling a liquid) By now the temperature must/will be at least / at most $15^{\circ}$, perhaps even $12^{0}$.

(30) By the year 2030 the world's top athletes will run a mile in at least / at most 4 minutes and 50 seconds, maybe even less.

These three examples differ from the ones discussed in the last section inasmuch as they involve ongoing movement along a scale. For (28) there is an implicit comparison between packing one's belongings into different cases, with the smallest one as the preferred option. In (30) the scale is not an athlete's actual performance now or in the past, but rather the expected process of constant improvement in athletic performance. Notice that all three examples include universal modals, denoting prediction.

The next example has a universal deontic modal:

(31) To qualify for the race you have to run a mile in at least / at most 5 minutes in the preliminaries.

Modality seems to facilitate this use of at least - for reasons that are not clear to me. But my final example, provided by an athlete, indicates that it is not a necessary condition;

(32) Yesterday John ran a mile in at least / at most 5 minutes.

What facilitates the use of at least here (and may also play a part in (30) and (32)) is the fact that in measuring the time taken in running a mile one also measures speed, and this is of prime importance in any racing context.

Recall that on a rising scale at least strengthens a claim, whereas at most weakens. At the beginning of this section I pointed out that on descending scales at most can strengthen but at least cannot, normally, weaken. The data discussed above indicate a fundamental asymmetry between these superlative modifiers. They are in competition for the same bit of meaning, with at least often the preferred option as a means of strengthening a claim.

\section{In Adverbials for Future Events \\ Consider}

(33) The train will arrive in ten minutes / in ten minutes' time. 


\section{Anita Mittwoch}

Is this use of the in-adverbial related to its use as a container adverbial? A speaker who utters (33) at 9.50 is committed to (34):

The train will arrive at 10 .

Both sentences allow the modifiers in (35):

(35) a. The train will arrive in ten minutes at the earliest / at the latest.

b. The train will arrive at 10 at the earliest / at the latest.

It would seem that the adverbial here focuses on a specific time at the end of ten minutes, just as the (postpositional) PP in (36) focuses on a specific time at the beginning of a ten-minute interval.

The train arrived ten minutes ago.

Both forms are deictic; the ten-minute interval is measured from speech time. ${ }^{10}$ Haspelmath (1997) classifies both as temporal distance adverbials.

On the other hand, Kearns (2003:605) treats the construction in (33) as a special case of what she calls a 'delayed onset reading' of in-adverbials as in

She noticed the marks in five minutes. $\quad(=$ Kearns $(6 a))$

The inference from (33) to (34) in the context given would be an implicature. In support of the connection between this construction and what I have called container adverbials I might point out that for me and other speakers I have consulted the restrictions on adverbials are operative here in the same way as we saw in section 2, which suggests an implication of a low value on the scale. We feel comfortable with (38a) but not with (38b):

(38) a. The train will arrive in less than/ at most / only ten minutes.

b. The train will arrive in more than / at least ten minutes.

Kearns does not specify the type of implicature that she has in mind, but her discussion of in-adverbials in general suggests that she means a conversational implicature. In my view if it is an implicature, it would have to be a conventional one.

On balance, I am inclined to side with Haspelmath, and to regard the construction as a future 'distance' adverbial. Thus the inference from (33) to (34) in the context would be truth-conditional. However, pragmatically the future distance

\footnotetext{
${ }^{10}$ For some speakers in ten minutes in this sense can have a contextually given past reference point, with would instead of will - in contexts other than Free Indirect Discourse. In FID contexts ago can also have a past reference point.
} 


\section{Numeral Modifiers and Container Adverbials}

adverbial would differ from its past counterpart inasmuch as it shares the evaluative implication of container adverbials, which means that the scale along which it measures would have to be a descending one.

I conclude this section with some examples from Google showing that by no means all speakers reject at least in the construction discussed here:

a. I have finished the first and am about to start on the third... which will be out in at least two years' time.

b. Also - we are hoping to get married in at least two years' time.

c. I'm sure he'll be back in at least a year's time.

d. The only way out is to draft a temporary one now, and aim for the final one in at least two years' time.

I think someone will achieve it, but in at least a year's time.

Judging by the content of the examples in (39), the speakers use at least in the sense of 'at most', i.e. the $\leq$ relation. This recalls the use of at least that we saw in the previous section; it strengthens the speaker's claim. Possibly, it is connected to the affinity between future and modality.

In (40) the use of but suggests that the speaker means the $\geq$ relation. I would guess that for such a speaker in a year's time is a true mirror image of a year ago with 'a year' being a value on a rising scale.

\section{Conclusion}

This paper has shown that the use of numeral modifiers is heavily dependent on speakers' evaluative assumptions about the numbers involved in what is being counted or measured. The ensuing restrictions, it was argued, are due to purely rhetorical reasons. It was then shown that the restrictions associated with a speaker's low estimate of a numerical value are systematically found in container adverbials, which indirectly measure the temporal length of an extended telic event by measuring the interval that exhaustively contains it. This finding is attributed to an evaluative meaning component of these adverbials, an inbuilt bias towards a low value and a fast event - or, at any rate, against a high value and a slow event. Additionally, the paper discusses a curious asymmetry between at least and at most, which shows up both in container adverbials and in future in-adverbials.

\section{References}

Beck, Sigrid, and Hotze Rullmann. 1999. A flexible approach to exhaustivity in questions. Natural Language Semantics 7(3):249-297.

Fox, Danny, and Martin Hack1. 2006. The universal density of measurement. Linguistics and Philosophy 29(5):537-586.

Grice, H. Paul. 1975. Logic and conversation. In P. Cole, and J.L. Morgan, eds., Syntax and semantics vol. 3: Speech acts, 41-58. New York: Academic Press. 
Haspelmath, Martin. 1997. From space to time: Temporal adverbials in the world's languages. Munich, Newcastle: Lincom Europa.

Horn, Laurence. 1972. On the semantic properties of logical operators in English. Ph.D. diss., University of California, Los Angeles.

Horn, Laurence. 2002. Assertoric inertia and NPI licensing, Proceedings of Chicago Linguistic Society 38-2, The Panels, 55-82. Chicago: Chicago Linguistic Society.

Jespersen, Otto. 1949. Syntax. Part 2 of A modern English grammar on historical principles. London: Allen \& Unwin.

Kearns, Kate. 2003. Durative achievements and individual-level predicates on events. Linguistics and Philosophy 26(5):595-635

Krifka, Manfred. 1998. The origins of telicity. In S. Rothstein, ed., Events and grammar, 197-236. Dordrecht: Kluwer.

Krifka, Manfred. 2006. Three, more than two, at least three: What's the difference? Handout downloadable from amor.rz.hu-berlin.de/ h2816i3x/.

Krifka, Manfred. 2007. More on the difference between more than two and at least three. Handout downloadable from amor.rz.hu-berlin.de/ h2816i3x/.

Mittwoch, Anita. 1980. The grammar of duration. Studies in Language 4(2):101227.

Mittwoch, Anita. 2010. Event measurement and containment. In M. Rappaport Hovav, E. Doron, and I. Sichel, eds., Syntax, lexical semantics, and event structure, 252-266. Oxford: Oxford University Press.

Nouwen, Rick. 2006. Remarks on the polar orientation of almost. In J. van de Weijer, and B. Los, eds., Linguistics in the Netherlands, 162-173. Amsterdam; Benjamins.

Nouwen, Rick. 2008. Upper-bounded no more: The exhaustive interpretation of non-strict comparison. Natural Language Semantics 16:271-295.

Stoffel, C. 1894. Studies in English. Zutphen: W.J. Thieme \& Co.

Department of English

The Hebrew University of Jerusalem

Mount Scopus

Jerusalem 91905

Israel

msanita@mscc.huji.ac.il 